\title{
The influence of selective attention in peripheral and foveal vision
}

\author{
BRUCE A. AMBLER and DIANNE L. FINKLEA \\ University of Texas at Arlington, Arlington, Texas 76019
}

\begin{abstract}
Previous research had indicated that there were differences in the kinds of stimulus information processed by concentrated and distributed attention in peripheral vision. Concentrated attention was necessary for perceiving line arrangement differences, while line slant differences could be detected with distributed attention. However, experiments with foveal presentation showed no facilitation by concentrating attention. Experiment I replicated these results. Experiment II showed that distributed attention did degrade foveal line arrangement discrimination if the attention system was overloaded by increasing the number of elements in the array. Experiment III demonstrated that high element density was not a sufficient condition for these effects to occur. Line arrangement discrimination was reduced as letter number increased even when maximum density was held constant. In Experiment IV, it was shown that concentrating attention facilitated line arrangement discrimination relative to line slant discrimination in foveal vision when element number and density were held constant. The results are discussed in terms of several models postulating a difference between spread-out and concentrated attention systems.
\end{abstract}

Beck and Ambler $(1972,1973)$ have investigated the processing involved in the discrimination of figures on the basis of differences in line slant and line arrangement. Only line slant differences are involved in the discrimination of a tilted $T$ from an upright $T$. The two lines which make up the figures are in the same arrangement relative to each other for both figures. The upright and tilted $\mathrm{T}$ differ only in the slant of the component lines. The discrimination of an $L$ from an upright $\mathrm{T}$ involves only the detection of differences in line arrangement. Although both figures contain only horizontal and vertical lines, the arrangement of these lines relative to each other differ between the two figures.

In conditions of position uncertainty, i.e., distributed attention, an $\mathrm{L}$ was more difficult to discriminate than a tilted T. However, as attention was directed toward the critical letter, performance on the T-L discrimination improved while no appreciable effect was shown on the T-tilted-T discrimination. Beck and Ambler (1973) proposed that attending to the critical element allowed a more complete analysis, facilitating observation of line arrangement differences. This was not necessary for perception of line slant differences. As attention is spread out, the processing system has enough capacity to analyze many elements simultaneously for line slant differences, but not for line arrangement differences. For discriminations based on line arrangement differ-

Requests for reprints should be sent to Bruce A. Ambler, Department of Psychology, University of Texas at Arlington, Arlington, Texas 76019. This research was supported by funds provided by the organized Research Committee of the University of Texas at Arlington. D. L. Finklea is now at the Institute of Behavioral Research, Texas Christian University, Fort Worth, Texas 76129. ences, the analysis of the elements in a multielement array cannot go on at the same time. Rather, the capacity of the attention system is allocated sequentially to some subset of elements. If no information about the critical location for an analysis is provided, the attention system must sample sequentially until the difference is found or it runs out of time. It appears that the number of elements and/or the area processed may be much larger for discrimination of line slant differences than for line arrangement differences. In searching for line slant differences, more parallel and less sequential processing may take place than in the search for line relationship differences.

At least two different models of visual attention have been presented which propose that attention can be spread out or concentrated. Eriksen and Rohrbaugh (1970) proposed that attention may be compared to a variable power lens. Distributed attention is analagous to a lens at a low power setting. Many elements in the field are visible, but fine detail cannot be discriminated. As attention is concentrated, the viewing field narrows, and, as with a lens at a high power setting, there is a large increase in resolvable detail. Niesser (1967) proposed two different attention systems rather than two forms of one system. He differentiated between preattentive processes and focused attention. Preattentive processes analyze many elements at once over a large area, while focused attention analyzes fewer elements in a smaller area. The Beck and Ambler results are consistent with both these models. Line slant differences are picked up by spread-out or preattentive processes, while differences in line arrangement are picked up by focused attention. These ideas have been discussed recently by Norman and Bobrow (1975). They label limitations on discrimination when attention can be con- 

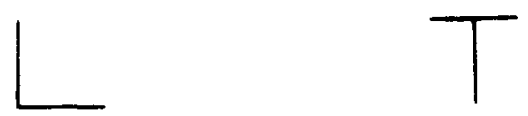

-

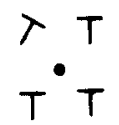

THMrRA

FOVEN

Figure 1. The relative position and size of stimuli used in the peripheral and foveal four-letter conditions in Experiment I. For these examples, an $\mathbf{L}$ is the disparate figure in the peripheral condition while a tilted-T is shown in the foveal condition. The one-letter condition used the same positions and sizes, but only one letter was presented.

centrated as a data limitation, while they call the limitation when attention is spread out a resource limitation. They refer to the idea that, when attention is concentrated on only one element, all the resources or capacity of the analysis system are allocated and any limitation on processing has to be due to the data which is provided to the system. However, when attention is distributed, discriminability is also influenced by the capacity allocated to each item to be analyzed. Thus, the analysis is limited by the resources available.

Beck and Ambler showed line arrangement differences to require concentrated attention and a sequential analysis of the visual array. However, there is some evidence that even for detecting differences in line arrangement the processing is done in parallel rather than sequentially.

Shiffrin and Gardner (1972) have produced evidence which failed to show that sequential analysis is involved in visual processing. Four figures were presented for discrimination, simultaneously or sequentially, with a 50-msec pause between presentations. It was expected that the sequential condition would result in increased target detection as attention could be shifted to each letter when it appeared. However, no difference in detection accuracy between simultaneous and sequential presentation was observed. This finding was particularly interesting because some of the discriminations involved only line arrangement differences. Beck and Ambler (1973) had clearly shown that concentration of attention on one element at a time facilitated discrimination performance for line arrangement differences.

There were several procedural differences between the two sets of experiments. The major difference appeared to be that, while Beck and Ambler presented their stimuli in peripheral vision, Shiffrin and Gardner presented their stimuli in the fovea. A comparison of the Beck and Ambler and the Shiffrin and Gardner experiments suggests that concentrated attention and sequential processing are necessary to detect line arrangement differences only in the periphery and not in the fovea.

The following experiments were designed to investigate the role of attentional processes in the discriminability of line slant and line arrangement differences for foveal and peripheral vision.

\section{EXPERIMENT I}

\section{Method}

Subjects. The subjects were eight university students whose participation in the experiment satisfied an introductory psychology course requirement. All subjects had normal vision without correction.

Apparatus and stimuli. Stimuli were presented in a Gerbrands three-field tachistoscope at a viewing distance of $81 \mathrm{~cm}$. They were drawn with a rapidograph No 2 pen in black ink on white cardboard.

The stimulus letters were upright $T$, and $L$ and $T$ tilted $30^{\circ}$ clockwise from vertical. The letters were constructed with two equal-length straight lines subtending visual angles of $47^{\prime}$ (foveal) and $1^{\circ} 47^{\prime}$ (peripheral). The difference in line length was made to control peripheral-foveal acuity differences. Acuity at $7^{\circ}$ is approximately 30\% that of the fovea (Christman, 1971, p. 139). The letters were located on the four corners of an imaginary square with a $7^{\prime}$ black dot as a central fixation point. Figure 1 shows the four letter positions and relative letter size for peripheral and foveal stimuli. In the foveal condition, the center point of each letter was located $47^{\prime}$ from the fixation point, and $6^{\circ} 46^{\prime}$ in the peripheral condition. The luminances of the tachistoscope fields were $.046 \mathrm{dd} / \mathrm{m}^{2}$ for the fixation field, $.80 \mathrm{~cd} / \mathrm{m}^{2}$ for the stimulus field, and $6.4 \mathrm{~cd} / \mathrm{m}^{2}$ for the blank field. The average luminance of the experimental room was $.47 \mathrm{~cd} / \mathrm{m}^{2}$.

Procedure. The subjects were allowed approximately $3 \mathrm{~min}$ for dark adaptation while instructions were read and questions answered. Each subject participated in three 1-h sessions, the first of which consisted of practice trials for task familiarization and establishment of exposure duration. The two following sessions were devoted to experimental conditions.

There were eight experimental conditions, four each for concentrated and distributed attention. Discriminations were T-L or T-tilted-T in one- and four-letter conditions, presented foveally and peripherally. In the concentrated attention condition, a single letter was presented in one of the four corners of the foveal or peripheral square. For distributed attention, four letters were presented simultaneously, one each in the corners of the peripheral or foveal square. These letters were either all upright Ts or three $T s$ and the disparate letter ( $L$ or tilted- $T$ ). On any block of trials, either the T-L or T-tilted-T discrimination was to be made. Sequence and letter position for each block of trials were randomized, with the disparate letter appearing equally often in each corner position. Within each block, half of the trials contained a disparate figure. The order of condition presentation was counterbalanced across subjects.

During the first session, exposure durations were established using the one-letter condition, T-L discrimination. An error rate of approximately $15 \%$ was allowed. For foveal presentation, exposure durations were $20-40 \mathrm{msec}$ (mean $=33 \mathrm{msec}$ ), and for peripheral, $34-56 \mathrm{msec}$ (mean $=47 \mathrm{msec}$ ). Generally, exposure durations were $10-15 \mathrm{msec}$ faster for foveal than for peripheral conditions. 
Experimental days were begun with five warm-up trials in the initial condition to be presented, at the previously established exposure duration. On each of the experimental days, only foveal or peripheral presentations were made. Subjects depressed a lever to initiate each trial, which presented first the fixation point for $500 \mathrm{msec}$, followed by the stimulus field and finally a 2 -sec blank field.

Following the warm-up trials, the subjects were run in the experimental conditions. Each condition was run for two blocks of 16 trials, resulting in a total of 32 trials per condition. Half of the subjects received the foveal conditions on the first experimental day, while the rest saw the peripheral figures. One block of each of the four letter type by letter number combinations was run, and then the sequence was repeated. However, the order of disparate figure blocks was reversed for the second set of blocks. The order of yes and no trials was randomized, and the disparate figures occurred equally often in each corner of the square in each block. The subjects were informed about the type of discrimination to be made prior to the initiation of each block of trials and were given feedback as to their accuracy following each trial.

\section{Results}

Mean errors for each condition were computed for all subjects. The means of these means are presented in Table 1 as percentages. Foveal and peripheral stimuli were analyzed separately using a two-way analysis of variance (Letter Type by Letter Number).

For the foveal condition, there were no significant differences. The effect of letter type resulted in an $F<1$, letter number produced an $F(1,7)=2.47$, $p<.05$, and the interaction had an $F<1$. The experiment replicated the earlier results of Shiffrin and Gardner in that neither experiment obtained significant differences between foveal presentations of one letter at a time and four simultaneous letters for discriminations of line arrangement differences. In the present experiment, however, there was a trend toward an effect of letter number and the possibility of such an effect cannot be ruled out. Also, there was no hint of an interaction, which made these results quite different from those previously obtained in the periphery. Beck and Ambler had found line slant differences to be more discriminable than line arrangement differences under conditions of distributed attention but not under conditions of concentrated attention. This interaction effect was not observed in the fovea.

It should be noted that Shiffrin and Gardner did find a significant difference between high and low confusion figures. In the present experiment, the slant of the tilted $\mathrm{T}$ was chosen so that it would be approximately equally confusable with the $T$ as an $L$ was in the one-letter foveal condition. This experiment was not analytic to overall confusability differences.

The results for the peripheral conditions were quite different. There was a significant effect of letter type, $F(1,7)=14.3, p<.001$, with Ls showing more errors than tilted Ts. There was also an effect of letter number, $F(1,7)=14.3, p<.001$, caused by the fact that there were more errors in the four-letter than in the one-letter condition. Most importantly, there was a significant
Table 1

Mean Percentage Errors: Experiment I

\begin{tabular}{lccccc}
\hline & \multicolumn{2}{c}{ One Letter } & & \multicolumn{2}{c}{ Four Letters } \\
\cline { 2 - 3 } \cline { 5 - 6 } & $\mathrm{L}$ & Tilted T & & L & Tilted T \\
\hline Fovea & 16.8 & 14.8 & & 21.5 & 18.4 \\
Periphery & 18.4 & 10.5 & & 33.2 & 15.6 \\
\hline
\end{tabular}

interaction effect, $F(1,7)=14.1, p<.001$. This interaction was due to the fact that the four-letter condition increased the difficulty for Ls much more than for the tilted $\mathrm{T}$ discrimination. This result replicated the previous finding of Beck and Ambler (1972) and Beck (1974).

The results in peripheral vision are consistent with the idea that concentrating attention on one letter facilitates processing, and that the concentration of attention is more beneficial to the processing of line arrangement differences than of line slant differences.

\section{Discussion}

It is tempting to conclude from these results that attention operates very differently in the fovea than in the periphery. The attention system for peripheral discrimination has a limited capacity, which must be more concentrated for discrimination of differences in line arrangement than for differences in line slant. In the fovea, however, the capacity of the system is unlimited and no differences exist in the degree of concentrated attention required for the two discriminations.

The problem with this conclusion is the assumption of unlimited capacity in the foveal attention system. It is possible that the capacity of the attention system in the fovea is greater than in the periphery, but not unlimited. Four letters may not have been enough to overload the capacity of the foveal attention system.

One way to attempt to overload the system would be to present it with more than four letters at once. In this way, it might be possible to show that for an overloaded foveal system the discrimination of the line arrangement differences requires a more concentrated attention system (and a more sequential analysis) than does the discrimination of line slant differences. Such a demonstration would indicate that the attention system in the fovea is not qualitatively different from the attention system in the periphery.

\section{EXPERIMENT II}

\section{Method}

Subjects. The subjects were seven university undergraduates whose participation fulfilled a course requirement. All had normal vision without correction.

Apparatus and stimuli. The apparatus was the same as that used in Experiment I, with the following exception: the fixation point was a light with a luminance of $3.8 \mathrm{~cd} / \mathrm{m}^{2}$ directed through a circular hole subtending a visual angle of $7^{\prime}$. This light was positioned to mark the center of the letter arrays while the remainder of the fixation field was dark. 


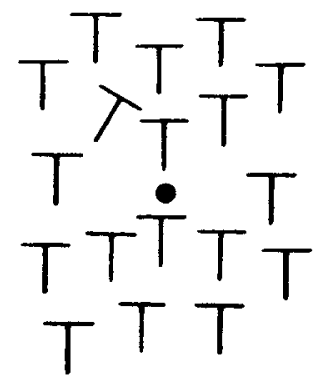

18 LETTERS

Figure 2. An example of an 18-tetter foveal stimulus used in Experiment II with a tilted-T as a disparate figure. The disparate figure could occur only in the four positions corresponding to the 4tetter foveal positions shown in Figure 1.

The stimuli were the 1- and 4tetter foveal stimuli from Experiment I. An additional set, containing 18 letters (shown in Figure 2), was constructed by the addition of 14 upright Ts to the positions surrounding the 4 letters in the foveal stimuli. These 14 letters acted only as additional elements, and disparate figures never occurred in any of the new letter positions. Position of occurrence of the disparate letters was exactly the same for the 1-, 4-, and 18-tetter conditions. That is, the critical positions for all conditions were the four corners of the foveal square shown in Figure 1. Noise letters were positioned such that the minimum distance between a letter in one of the four critical positions and a noise letter was 19'. Each critical position was surrounded by three noise letters, with the remaining letters positioned to present the appearance of an approximately uniform letter density.

Procedure. The procedure was similar to that used in Experiment $I$. The subjects were run in two 1-h sessions. During the first session, a minimum of 16 practice trials in all conditions were given. During this time, an exposure duration was established, allowing approximately a $20 \%$ error rate in the 1 -letter $L$ condition. The exposure durations used had a mean value of $30 \mathrm{msec}$, with a range of 25 to $39 \mathrm{msec}$. These exposure durations were used in the 1- and 4-letter conditions. For each subject, $5 \mathrm{msec}$ was added for the exposure durations of the 18letter condition in an attempt to prevent overall error rates from going too high in this condition. The subjects were then run in blocks of 24 trials for each letter type in all conditions. Order of presentation of stimulus conditions was counterbalanced across subjects as before. The subjects received two blocks of 24 trials in each letter discrimination in each condition.

\section{Results}

For each subject, the mean errors for the two blocks of trials for each condition were computed. The means of these means are presented in Table 2 as a percentage of total trials. The data were analyzed with a two-way analysis of variance (Letter Type by Letter Number). There was no significant effect of letter number, $F(2,12)=1.0, p<.05$. There was an effect of letter type, $F(1,6)=36.3, p<.01$. This appeared to be due to the $\mathrm{Ls}^{\prime}$ producing more errors than tilted Ts in the 18-letter condition. This interpretation is supported by the fact that there was a significant interaction effect, $F(2,12)=10.7, p<.05$. A post hoc Scheffé analysis for a within-subjects design was used to show where the interaction effect was occurring. The tilted $\mathrm{T}$ and $\mathrm{L}$ error rates did not differ significantly in either the 1-letter, $F(1,12)=1.31, p<.05$, or the 4-letter, $F<1$, conditions. However, in the 18-letter conditions, the tilted Ts produced significantly fewer errors than did the $\mathrm{L}, F(1,12)=9.18, p<.025$.

The rather large drop in error rate for tilted $T s$ in the 18-letter condition is probably due to the fact that the 18 letter condition had a 5 -msec longer exposure duration. In spite of this, performance on Ls grew worse and there was a significant difference between the two letter types.

\section{Discussion}

The results of Experiment I were replicated in that there was no significant difference in the ability to discriminate the line slant differences and the line arrangement differences in the fovea when 1 or 4 letters were presented. However, when 18 letters were presented, line slant differences were much easier to detect than were line relationship differences. This effect occurred only in the 18-letter condition, even though the disparate figures were being presented in exactly the same positions in all three conditions. These results demonstrate in the fovea the same differential performance for discrimination of line slant and line arrangement differences observed by Beck and Ambler (1972) in peripheral vision. However, this effect only showed up in the fovea when the attention system had been overloaded. The data from Experiments I and II indicate that, in the fovea, attention can analyze 4 letters about as well as 1 letter, even for line arrangement differences. There were no significant differences in error rate for either discrimination type across these conditions. However, the capacity of the system is not infinite. It cannot process 18 letters as well as 1 or 4 letters for differences in line arrangement. When the capacity of the attention system has to be distributed over 18 letters, then tilted Ts are more discriminable than Ls.

With the addition of more. letters in the 18-letter condition, actually two factors were being varied simultaneously. These were number of letters and letter density. Either one or both of these variables could be the factor which was overloading the attentional system.

The possible effect of density has to be considered more closely. Estes (1972) argued that changes in discriminability associated with changes in letter number were not necessarily due to a change in the distribution of attention. Adding more elements in an array results in more inhibition of other oloments in the array and, therefore, reduced performance.

Table 2

Mean Percentage Errors: Experiment II

\begin{tabular}{cccc} 
& \multicolumn{3}{c}{ Letter Number } \\
\cline { 2 - 4 } Letter Type & 1 & 4 & 18 \\
\hline Tilted T & 30 & 23 & 12 \\
L & 22 & 25 & 36 \\
\hline
\end{tabular}


According to the model, this reduction in ability to detect figures occurs when figures are close together and so compete for the same analyzer system. Thus, increasing density puts more elements in close proximity to one another and reduces performance. This model can explain the greater reduction in detection accuracy for the T-L discrimination by assuming that figures competing for the analysis of lines of the same orientation interfere with each other more than figures competing for the analysis of lines of different orientations.

This interference model and the attention model make very different predictions concerning which letters in the 18-letter array are causing the differences in accuracy between the line slant and line arrangement discrimination. The interference model predicts that the most important letters are those closest to the disparate figure. The same effect should be obtained by using only one critical figure and several noise figures very close to it. The other noise figures should not be important, because they are not competing with the critical figure for analysis.

The attention model predicts that it is the total number of figures which have to be analyzed that is producing the differences in discriminability. If only a few elements are clustered together in the field, then attention should be able to be focused on them and the effects of a distributed attention system should be reduced. When there is a uniform field of many elements the line-slant-line-arrangement discriminability differences should be observed. Under these conditions, the attention system is distributed and overloaded.

Experiment III was conducted in an attempt to separate the influence of letter density and number on the discriminability differences observed in Experiment II.

\section{EXPERIMENT III}

\section{Method}

Subjects. The subjects were 15 university students who received credit toward a course requirement for participation. All had nermal vision without correction.

Apparatus and stimuli. The apparatus was the same as in Experiment I. Two of the stimulus conditions were replications

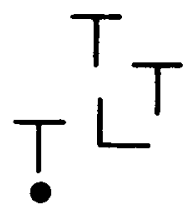

\section{DENSE LETTERS}

Figure 3. An example of a stimulus used in the four-dense condition in Experiment III with an $L$ as a disparate figure. The positions of the disparate figures were the same as those in the 18-tetter condition (Figure 2). The position of the three upright $T$ noise letters closest to the disparate figure in the 18-letter condition were used as the positions for the three-noise letters in the four-dense condition.
Table 3

Mean Percentage Errors: Experiment III

\begin{tabular}{lccc} 
& \multicolumn{3}{c}{ Letter Number } \\
\cline { 2 - 4 } Letter Type & 4 & 4 Dense & 18 \\
\hline Tilted T & 16.5 & 20.2 & 17.2 \\
L & 18.9 & 27.9 & 33.1 \\
\hline
\end{tabular}

of the 4- and 18-letter conditions of Experiment II. Stimuli for the four-dense condition (Figure 3) were made from the stimulus display used in the 18-letter conditions. For each of the four locations where a disparate figure could occur, Ts were placed around the critical position such that they correspond to the location of the 3 closest letters in the 18-tetter condition. In the center of these $T$ s, there was either another upright $T$ or a disparate figure. For the four-dense condition, each stimulus card contained 4 letters occupying the same positions as 1 disparate letter and its three nearest neighbors in the 18 tetter condition. The maximum density was therefore the same for these two conditions. However, in the four-dense condition, there were only 4 letters clustered together, so that attention could be shifted and focused on the area where the critical letter could occur. This shift in attention might not be as accurate as in a 1-letter condition, but it should be better than the 18-letter condition.

Procedure. The procedure used was the same as that used in Experiment 1I, except that when an exposure duration was established for each subject, it was used for all three conditions. The exposure durations had a mean value of $40 \mathrm{msec}$ and a range of 30 to $55 \mathrm{msec}$.

\section{Results}

The mean percent errors for each condition are presented in Table 3. The data were analyzed with two separate analyses of variance. The first analysis considers the effect of density while number is controlled, by comparing the four to the four-dense condition. A twoway analysis of variance (Letter Type by Letter Density) did show a significant effect of letter type, $F(1,14)=7.8, p<.025$. Error rate was lower for tilted Ts. There was also a significant effect of density, $F(1,14)=9.1, p<.01$. Error rate for both types of discriminations was greater in the four-dense condition. However, density alone did not produce a significant interaction, $F(1,14)=4.0, p<.05$, when attention could be shifted.

Another analysis was performed comparing the fourdense and the 18-letter conditions. In this comparison, maximum density is the same for both conditions, but number of letters is different. The two-way analysis of variance (Letter Type by Letter Number) resulted in a significant effect of letter type, $F(1,14)=44.0$, $p<.01$, with Ls resulting in more errors. There was no main effect of letter number, $F<1$. However, there was a significant interaction, $F(1,14)=5.6, p<.05$. Increasing the number of letters from 4 to 18 resulted in an improvement in performance for the tilted Ts, while it decreased performance on the Ls.

\section{Discussion}

The increase in density while holding number constant did result in an increase in error rate. These results indicate that increasing density degrades performance. There are at least two possible reasons why performance was not degraded significantly more in line arrange- 
Table 4

Mean Percentage Errors: Experiment IV

\begin{tabular}{ccc}
\hline & \multicolumn{2}{c}{ Attention Condition } \\
\cline { 2 - 3 } Letter Type & Spread-Out & Concentrated \\
\hline Tilted T & 7 & 13 \\
L & 29 & 22 \\
\hline
\end{tabular}

ment discriminations than in line slant discriminations. First, degradation due to density increases may not be related to line slant differences. This would mean that density does not contribute at all to the differences observed in the 18-letter condition. However, it may also be that increasing letter density using letters with vertical and horizontal lines interferes more with the T-L discrimination. In the four-dense condition, attention can be concentrated on the critical position and this overcomes the differential effect of density. At this time, these two interpretations cannot be differentiated.

The results do show that density alone is not a sufficient condition to have a differential effect on line slant and line arrangement discriminations. Only in the 18-letter condition, where letter number is increased and attention cannot be concentrated, is the T-L discrimination degraded while T-tilted-T discrimination is not. This suggests that overloading the attention system is necessary in order to produce the effect.

One objection which might be raised to this conclusion is that when noise elements are added to an array, it is not the near elements which are having the major effect, but rather the elements which are farther away from the disparate figures. In controlling for maximum density, the effects of these elements were not observed.

Experiment IV was conducted to see if a differential effect of concentrated and distributed attention could be demonstrated when all 18 letters were in the array.

\section{EXPERMENT IV}

\section{Method}

Subjects. The subjects were seven university students who had normal vision without correction. All of the subjects received credit toward a course experimental participation requirement. One other person was run for a practice session. However, he was not continued in the experiment because he reported that he could not consistently see the cuing dots when they occurred.

Apparatus and stimuli. The apparatus was the same as that used in Experiment I. The luminance values for the three fields were $.54 \mathrm{~cd} / \mathrm{m}^{2}$ (fixation), $.51 \mathrm{~cd} / \mathrm{m}^{2}$ (stimulus), and $1.46 \mathrm{~cd} / \mathrm{m}^{2}$ (blank).

The cards from the 18-tetter condition in the previous experiment were used as stimuli. Cuing dots were used to create a condition in which attention could be concentrated. These were black dots subtending an angle of $10^{\prime}$ on a white field. Each dot was positioned such that it fell on a straight line running through the fixation point and one of the four critical positions where a disparate figure could occur. The dot appeared along this line about $21^{\prime}$ outside the closest point of the critical figure.

The fixation point was on continuously and went off 500 msec after a trial was initiated by the subject. In the concentrated attention condition, after the fixation point went off, a cuing dot occurred for $50 \mathrm{msec}$. After this time, the cuing dot went off and the letter array came on. Although the cuing dot and letters did not occur simultaneously, they gave the appearance of occurring together. The mean exposure duration of the letters was $29 \mathrm{msec}$, with a range of $20-35 \mathrm{msec}$. The maximum total time for cuing dot and letter stimuli was $85 \mathrm{msec}$, which minimized any possible effect of eye movements.

In the spread-out attention condition, a blank white card was substituted for the cuing dot card but the field presentation sequence remained the same.

Procedure. The procedure was similar to that of previous experiments. The subjects were run in two $1-\mathrm{h}$ sessions. During the first half of the first session, the subjects received practice in all conditions. They were then run in each condition if blocks of 24 trials. For each letter discrimination, the subjects were run in two blocks of trials in each condition. The order of discrimination type was counterbalanced across blocks. In the cuing dot condition, the subjects were given instructions concerning the dot's location and function. They were informed that the dot would mark the position where a disparate figure would occur if it was going to be present and that attention should be directed to the area where the cuing dot was presented.

\section{Results}

The mean percent errors are presented in Table 4. The data were analyzed with a two-way analysis of variance (Letter Type by Attention Condition). There was a significant effect of letter type, $F(1,6)=23.6, p<.01$. Accuracy was less for $L s$ than for tilted Ts. There was no main effect of attention condition, $F<1$. A significant interaction was obtained, $F(1,6)=12.1, p<.025$. When the subjects used the cuing dot to concentrate their attention on the position where a disparate figure could occur, their performance on the line relationship discrimination was improved. However, concentrated attention appeared to reduce performance on the line slant difference discrimination.

\section{Discussion}

The results replicate for foveal presentation the basic finding of Beck and Ambler (1973). For foveal presentation, allowing attention to be concentrated improved the ability to discriminate line relationship differences. It had the opposite effect on line slant discrimination.

These results show an improvement in performance on line relationship discrimination without changing the letter array. Removal of the distant noise elements in the four-dense condition of Experiment III is not a necessary condition for line relationship discrimination improvement.

The best way to explain these results is to assume that allowing subjects to concentrate attention facilitated performance in discrimination of line relationship differences. One reason why the effect was not as large as those previously observed is that the $50 \mathrm{msec}$ during which cuing dots were presented is probably not susficient time to fully concentrate attention.

\section{SUMMARY AND CONCLUSION}

Differences were observed between visual attention in peripheral and foveal vision. In peripheral vision, 
when attention was changed from being concentrated on one figure to being distributed over four figures, there was a larger decrease in discriminability for line arrangement differences than there was for line slant differences. However, no significant changes in discriminability were observed between the one- and four-letter conditions in foveal vision.

We proposed that 4 letters did not sufficiently overload the capacity of the attention system in the fovea. When the letter array contained 18 letters, the attention system was overloaded and large differences were observed between the line slant and line arrangement discrimination. The discriminability of line arrangement differences was reduced in peripheral vision with only 4 letters. However, in foveal vision this reduction in discriminability occurred with 18 letters. This indicates that the capacity of the attention system is more easily overloaded in peripheral vision than in foveal vision. Increasing letter number did not appear to degrade the discriminability of foveal line slant differences, indicating that concentrated attention was not necessary for such processing and the detection of line slánt differences could occur with a distributed attention system.

Within foveal vision, recent evidence indicates that there is a maximum degree to which attention can be concentrated (Eriksen \& Eriksen, 1974; Eriksen \& Hoffman, 1973). Eriksen found, for example, that adjacent noise elements were processed even when 350 msec was allowed to concentrate attention on one letter position. As attention is concentrated on a smaller and smaller area of the visual field, a point may be reached where the attention system has the capacity to process simultaneously all information in that area, even for line arrangement differences. However, the fovea is not such an area.

The present experiments showed that increasing the number of letters in the foveal array had its effect by influencing the attention system. A differential influence on the detection of line slant and line arrangement differences was not observed when attention could be concentrated on a smaller high-density array. The difference between line slant and line arrangement discriminations was reduced in the 18-letter array when a cuing dot allowed concentration of attention in one area of the array. High density was not a sufficient condition to overload the attention system.

The data from these experiments support the existence of two functionally different attention systems. The distributed system is much more sensitive to line slant differences than to line arrangement differences under conditions of high figure density. Whether these are two different systems (Neisser, 1967) or two different forms of the same system (Eriksen \& Rohrbaugh, 1970) cannot be determined at this time.

Experimenters studying visual attention and visual search should be careful about what kinds of discriminations are involved before generalizing their results. For example, when the discriminations involve detecting line slant difference, the attention system will appear to involve parallel processing. Performance will not vary much with the number of elements in the array. However, if the discriminations involve looking for line relationship differences, the system will appear much more like a serial process.

Discriminations cannot simply be characterized as being high or low in confusability. Two discriminations might be equally confusable under conditions of concentrated attention and yet be very different in their relative difficulty under conditions of distributed attention.

\section{REFERENCES}

BECK, J. Relation between similarity grouping and peripheral discriminability. Joumal of Experimental Psychology, $1974,102,1145-1147$.

BeCK, J., \& AMBLER, B. Discriminability of differences in line slope and in line arrangement as a function of mask delay. Perception \& Psychophysics, 1972, 12, 33-38.

BECK, J., \& AMBLER, B. The effects of concentrated and distributed attention on peripheral acuity. Perception \& Psychophysics, 1973, 14, 225-230.

Christman, R. Sensory experience. Toronto: Intext Educational Publishers, 1971.

ERIKSEN, B., \& ERIKSEN, C. Effects of noise letters upon the identification of a target letter in a nonsearch task. Perception \& Psychophysics, 1974, 14, 143-149.

Eriksen, C., \& Hoffman, J. The extent of processing of noise elements during selective encoding from visual displays. Perception \& Psychophysics, 1973, 14, 155-160.

Eriksen, C., \& Rohrbaugh, J. Some factors determining efficiency of selective attention. American Journal of Psychology, 1970, 83, 330-342.

Estes, W. Interactions of signal and background variables in visual processing. Perception \& Psychophysics, 1972, 12, 278-286.

Niesser, U. Cognitive psychology. New York: AppletonCentury-Crofts, 1967.

Norman, D., \& Bobrow, D. On data-limited and resourcelimited processes. Cognitive Psychology, 1975, 7, 44-64,

Shiffrin, R. M., \& Gardner, G. T. Visual processing capacity and attentional control. Journal of Experimental Psychology, 1972, 93, 72-82.

(Received for publication December 5, 1975; revision accepted March 22, 1976.) 